\title{
High-spatial-resolution ultrafast framing imaging at 15 trillion frames per second by optical parametric amplification
}

\author{
Xuanke Zeng, ${ }^{a, b}$ Shuiqin Zheng, ${ }^{a}$ Yi Cai, ${ }^{a}$ Qinggang Lin, ${ }^{a}$ Jinyang Liang, ${ }^{c}$ Xiaowei Lu, ${ }^{a}$ Jingzhen Li, ${ }^{a}$ \\ Weixin Xie, ${ }^{b}$ and Shixiang $\mathrm{Xu}^{\mathrm{a}, *}$ \\ ashenzhen University, College of Physics and Optoelectronic Engineering, Shenzhen Key Lab of Micro-Nano Photonic Information Technology, \\ Shenzhen, China \\ bShenzhen University, College of Electronic Information Engineering, Shenzhen, China \\ 'Institut National de la Recherche Scientifique, Centre Énergie Matériaux Télécommunications, Laboratory of Applied Computational Imaging, \\ Varennes, Québec, Canada
}

\begin{abstract}
We report a framing imaging based on noncollinear optical parametric amplification (NCOPA), named FINCOPA, which applies NCOPA for the first time to single-shot ultrafast optical imaging. In an experiment targeting a laser-induced air plasma grating, FINCOPA achieved 50 fs-resolved optical imaging with a spatial resolution of $\sim 83 \mathrm{lp} / \mathrm{mm}$ and an effective frame rate of 10 trillion frames per second (Tfps). It has also successfully visualized an ultrafast rotating optical field with an effective frame rate of 15 Tfps. FINCOPA has simultaneously a femtosecond-level temporal resolution and frame interval and a micrometer-level spatial resolution. Combining outstanding spatial and temporal resolutions with an ultrahigh frame rate, FINCOPA will contribute to high-spatiotemporal resolution observations of ultrafast transient events, such as atomic or molecular dynamics in photonic materials, plasma physics, and laser inertial-confinement fusion.
\end{abstract}

Keywords: ultrafast imaging; spatiotemporal resolution; frame rate; noncollinear optical parametric amplification.

Received May 4, 2020; revised manuscript received Aug. 21, 2020; accepted for publication Aug. 24, 2020; published online Sep. 16, 2020.

(C) The Authors. Published by SPIE and CLP under a Creative Commons Attribution 4.0 Unported License. Distribution or reproduction of this work in whole or in part requires full attribution of the original publication, including its DOI.

[DOI: 10.1117/1.AP.2.5.056002]

\section{Introduction}

It is invaluable to discover and understand ultrafast dynamic processes, e.g., femtosecond laser ablation for precise machining and manufacturing, ${ }^{1}$ fast ignition for inertial-confinement fusion, ${ }^{2}$ laser filaments for atmospheric analysis, ${ }^{3}$ shockwave interactions in living cells, ${ }^{4}$ chemical reactions and soliton molecule dynamics, ${ }^{5-7}$ semiconductor thermal dynamics, and photosynthetic light harvesting. ${ }^{4,8}$ As a result, ultrafast optical imaging, which can realize blur-free visualization of transient dynamics, has attracted considerable attention for decades from researchers in many fields, such as physics, chemistry, optical engineering, industrial manufacturing, energy, materials science, and biomedicine. ${ }^{9}$ Time-resolved imaging based on the pump-

*Address all correspondence to Shixiang Xu, E-mail: shxxu@szu.edu.cn probe methods ${ }^{10,11}$ works very well for reproducible transient dynamics with a high repetition rate because its sampling interval (down to attoseconds) and temporal resolution are on the order of femtoseconds. However, this approach fails to work for events that either have a low repetition rate or are nonrepeatable.

Many methods for single-shot optical imaging ${ }^{12,13}$ have been developed to replace the pump-probe methods. Traditional highspeed cameras are limited by the response speed of electronics. A rate of 25 million frames per second (Mfps) has been obtained with some film-based cameras. ${ }^{14}$ Streak cameras have a temporal resolution at the femtosecond level but an unsatisfactory spatial resolution. ${ }^{15}$ Compressed ultrafast photography (CUP) ${ }^{16}$ can work at a frame rate of 0.1 trillion frames per second (Tfps) with a temporal resolution of $\sim 50$ ps by applying a compressedsensing-based algorithm to the data acquired by a streak camera, and its spatial resolution can be scaled to $7 \mathrm{lp} / \mathrm{mm} .{ }^{15}$ With the 
help of a $20 \times$ microscopic objective lens, the phase-sensitive CUP (pCUP) system ${ }^{17}$ can get a spatial resolution of a few micrometers and an imaging speed of 1 Tfps. To further improve the imaging speed, trillion-frame-per-second CUP (T-CUP) ${ }^{18}$ has reached a frame rate of 10 Tfps. However, limited by the corresponding temporal resolution ( $0.58 \mathrm{ps}$ for T-CUP or $7.6 \mathrm{ps}$ for pCUP), the effective frame rate is limited to $1.75 \mathrm{Tfps}$ for T-CUP or 0.135 Tfps for pCUP. These CUP-based devices do not need any other illuminating source and can work with a larger number of frames. However, it is a challenge for them to synchronize temporally the ultrafast event to the sampling signal, due to the passive operation mode, especially when the required frame rate is beyond Tfps.

All-optical single-shot ultrafast imaging methods have been developed to avoid bottlenecks arising from the response time of recording media and high-speed deflection/scanning devices. For example, serial time-encoded amplified imaging/ microscopy ${ }^{19}$ converting the spectrum of a pulse into a twodimensional (2D) rainbow using a disperser, can take 2D ultrafast continuous imaging at a frame rate of $\sim 6 \mathrm{Mfps}$ with a shutter speed of $440 \mathrm{ps}^{12}$ Frequency-domain tomography and multispectral tomography, mapping different spectral components of illumination onto different positions on the target, have captured single-shot tomographic images with a temporal resolution on the order of picoseconds. ${ }^{20,21}$ Sequentially timed all-optical mapping photography (STAMP) ${ }^{4}$ can get six frame images with a high pixel resolution $(450 \times 450$ pixels), while its frame rate can be up to $4.4 \mathrm{Tfps}$. The successive images are sampled by a series of discrete daughter pulses with different spectral bands generated from a single broadband pulse, so some constraints exist among the temporal resolution, frame interval, and frame number. ${ }^{422-24}$ By utilizing spectral filtering in STAMP, i.e., SF-STAMP ${ }^{24}$ the frame rate increases further to $7.5 \mathrm{Tfps}$ with a frame number of 25 , which also results in a sacrifice of the temporal resolution, at 465 fs. In this case, the highest effective frame rate is $\sim 2$ Tfps. Similar to STAMP, increasing the frame number in SF-STAMP will sacrifice the temporal resolution.

The frequency recognition algorithm for the multiple exposures (FRAME) technique ${ }^{25}$ works by attaching sinusoidal patterns onto illumination pulses for extracting the information of each frame image at the spatial frequency domain. It can produce four frames at $5 \mathrm{Tfps}$ with a spatial resolution of $15 \mathrm{lp} / \mathrm{mm}$. However, increasing its frame number will result in overlaps of information in the spatial frequency domain, which means that in FRAME a constraint exists between the frame number and spatial resolution. All-optical coaxial framing photography (AOCFP) $)^{26}$ can realize single-shot optical imaging for both phase and amplitude information with parallel coherence shutters. It has a frame rate of $8 \mathrm{Tfps}$ and a temporal resolution of about $40 \mathrm{fs}$, depending on the femtosecond pulse used..$^{27} \mathrm{~A}$ constraint also exists in AOCFP, where increasing the frame number will result in degradation of the interference contrast. Recently, by replacing electro-optical deflection with spectral shaping, an all-optical CUP named compressed ultrafast spectral-temporal photography ${ }^{28}$ has been realized, which can work with a frame rate of $3.85 \mathrm{Tfps}$ as well as a frame number of 40 . However, increasing its frame number also degrades the temporal resolution due to the spectral shaping. The time and spatial-frequency multiplexing imaging ${ }^{29}$ can take 14 snapshots with a frame rate of 5 Tfps and a 150 fs temporal resolution by the interference of reference pulses that have various propagation angles and time delays. However, the coaxial probe pulses and the off-axis reference pulses make it difficult to accurately synchronize the alignment between the probe and the reference pulses, along with the increase of the frame number. Until now, single-shot femtosecond imaging with a high-spatiotemporal resolution and a high frame rate/number has remained a great challenge.

Optical parametric amplification (OPA) is an attractive candidate to overcome limitations in existing single-shot ultrafast optical imaging. By applying OPA to optical imaging, ${ }^{30}$ information carried in the signal can be copied to the idler. This feature inspires us to develop a new single-shot ultrafast optical imaging method, termed framing imaging by noncollinear optical parametric amplification (NCOPA), or framing imaging based on noncollinear optical parametric amplification (FINCOPA). The noncollinear arrangement allows the conversion of information in the sequential frames into spatially separated idler images using multistage optical parametric amplifiers pumped by a sequential laser pulse train. Accordingly, FINCOPA records the images without the need for fast-response cameras. This all-optical method is also free from any bottleneck arising from active mechanical and electronic components for fast scanning or deflection, which is critical for high frame rates. As discussed later, the temporal resolution, spatial resolution, frame number, and frame interval are rather independent. In this regard, FINCOPA is a good candidate for single-shot imaging with high spatial and temporal resolutions, as it offers a high frame rate as well as a large frame number, simultaneously.

\section{Principle and System}

Figure 1(a) illustrates the principle of FINCOPA. We use a sampling pulse with a sufficiently long temporal width to cover all information in the targeted transient process. Additionally, a sequence of ultrashort pulses (marked as the trigger-1,2, 3, and 4) is used to trigger and switch the imaging information from different temporal slices of the sampling pulse to another sequence of ultrashort pulses (marked as the recorded-1, 2, 3, and 4) using cascaded optical imaging converters (i.e., OIC-1, 2, 3, and 4). Because the recorded images are spatially separated from each other, they can be received by different CCD cameras. The frame intervals are determined by the relative delays between the sampling pulse and the trigger pulses, whereas the exposure times of the images can be estimated using the trigger pulses' duration. Thus the exposure time, effective frame rate, and frame number do not depend on each other.

To implement this idea, a femtosecond laser system is required for femtosecond temporal resolution. Here accurate time synchronization is critical among the trigger pulses and the sampling pulse. We ensure the accurate synchronization by obtaining both the sampling pulse and the trigger pulses from the same laser source, which reduces the temporal jitters among the synchronized pulses to a few femtoseconds. ${ }^{31}$ The frame number of our imaging system, denoted by $N$, is determined by the ratio of the total available trigger pulse power to the required power to trigger each optical imaging converter.

OPA can map the signal information into the idler, so optical parametric amplifiers can serve as our image converters. Additionally, the use of ultrashort pulses as the pumps for OPA means a short exposure time in OPA imaging, i.e., a high temporal resolution. In an optical parametric amplifier, OPA only occurs during the interaction between the pump and signal, meaning that the image information is only mapped into the 

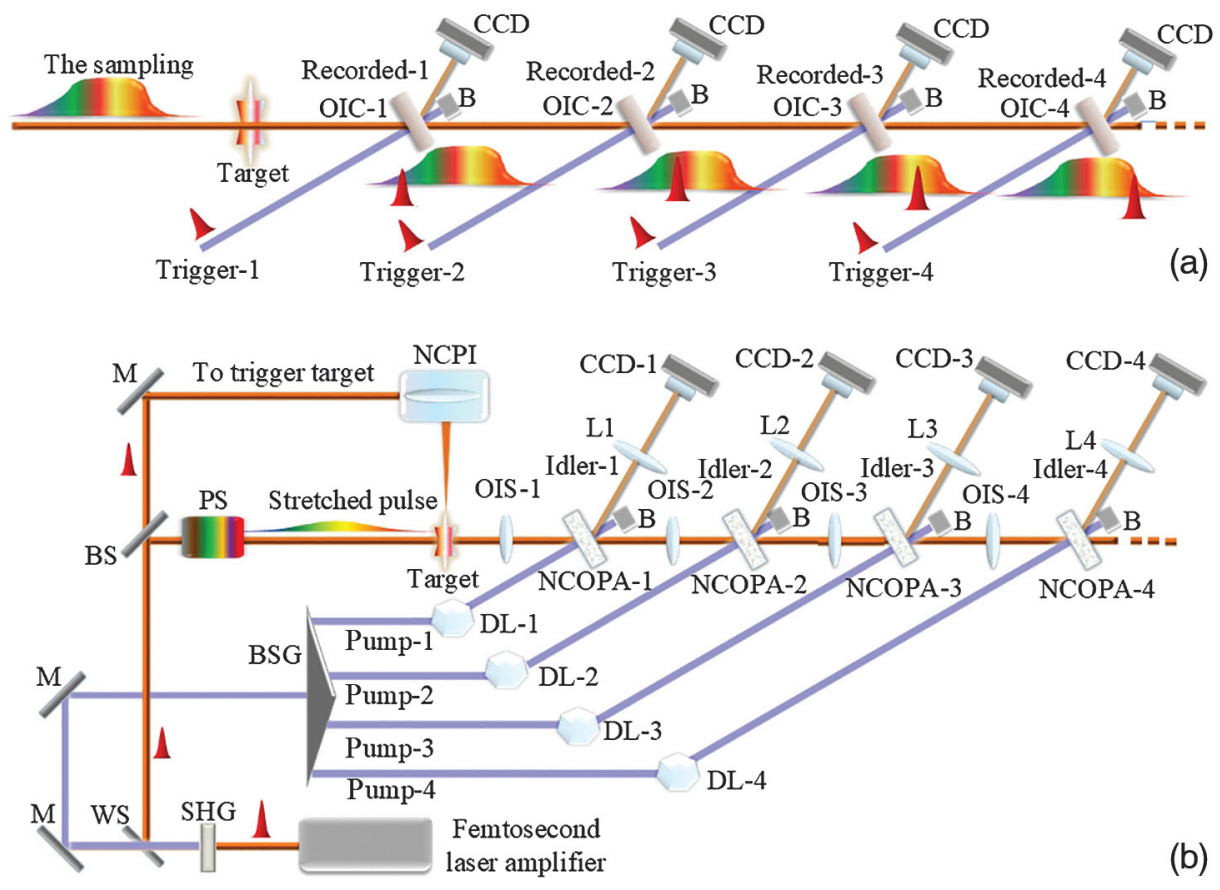

Fig. 1 (a) Schematic diagram and (b) experimental setup of FINCOPA. OIC-1 to OIC-4, optical imaging converters; B, barrier; M, mirror; BS, beam splitter; SHG, second-harmonic generator; WS, wavelength separator; NCPI, noncollinear plasma interferometer (details in the Appendix); OIS-1 to OIS-4, optical imaging systems; NCOPA-1 to NCOPA-4, noncollinear optical parametric amplifiers; DL-1 to DL-4, delay lines; BSG, beam splitter group; L1 to L4: lenses, CCD-1 to CCD-4, charge-coupled devices; and PS, pulse stretcher.

idler under irradiation by the pump. The pump has a much shorter pulse duration than the signal, so it can act as an optical shutter. The exposure time can be estimated by the pump pulse's duration, while the temporal resolution is mainly determined by the idler pulse's duration. Both durations are equal with each other if the thicknesses of the OPA crystals are designed to be sufficiently thin to suppress obvious temporal walk-off between the signal and the pump pulses. Moreover, the ultrashort duration of the pump pulse favors a high pump intensity (e.g., $\left.>100 \mathrm{GW} / \mathrm{cm}^{2}\right),{ }^{32}$ which is also helpful for high OPA gain and large time-space bandwidths. In other words, the pump intensity for OPA is determined by the required OPA gain and bandwidths but is also limited by the required imaging sizes and available pump power for OPA. For a given pump intensity and the imaging sizes or the pump area of each amplifier, the number of the amplifiers or the frame number can be estimated by the total pump power divided by the pump power of each amplifier. Furthermore, the delay time between the pump pulse and the signal pulse determines the exposed temporal slices of the signal in every amplifier denoted by $\tau$. From the differences among every value of $\tau$, we can determine the frame intervals. In the FINCOPA setup, $\tau$ is only limited by the minimum available step sizes of the time delay lines (DLs) and the fluctuations in the beam paths. Usually, the frame intervals are chosen to be larger than the pump pulse's duration. Otherwise, the effective frame rate should be estimated by the inverse of the pump pulse's duration instead of using the inverse of the frame interval.

To avoid the limitations in the response time of the recording media and the high-speed deflection/scanning devices, we get each of the idler images from a series of NCOPAs so that the idlers deviate spatially from both their own pumps and signals.
That is to say, the sequential frame images carried by different idler beams of the different amplifiers, are spatially separated, thus can be recorded with CCD cameras without the need for fast-response or fast-scanning components.

Figure 1(b) shows the experimental setup of FINCOPA. The used femtosecond laser source is a commercial $1 \mathrm{kHz}, 800 \mathrm{~nm}$ Ti:sapphire (Ti:S) laser system with a pulse duration of $\sim 40 \mathrm{fs}$ and an energy of $3.5 \mathrm{~mJ}$. Its output first passes through a secondharmonic generator (SHG), a $0.2-\mathrm{mm}$ and 29.2 deg-cut $\beta$-BBO crystal. Approximately $30 \%$ of the laser pulse is converted into its second harmonic (i.e., a 400-nm pulse) with a pulse duration of $\sim 40 \mathrm{fs}$. After passing through a wavelength separator (WS), the 400-nm pulse is split into four daughter pulses by a beam splitter group (BSG), including three 50:50 beam splitters (BSs), to pump four optical parametric amplifiers (NCOPA-1 to NCOPA-4). Here the number of the amplifiers or the frame number is four, which is mainly limited by the output pulse power of our femtosecond laser system $(\sim 3.5 \mathrm{~W}$ at $1 \mathrm{kHz})$. If the energy of the femtosecond laser reaches $7 \mathrm{~W}$, the frame number can be estimated at $4 \times 7 / 3.5=8$. The unconverted $800 \mathrm{~nm}$ fundamental pulse is reflected by the WS. About $1 \%$ of the 800-nm laser pulse is guided to a pulse stretcher (PS), a grating-based pulse disperser, which stretches the pulse duration up to $50 \mathrm{ps}$. The stretched pulse then works as the sampling to illuminate the targeted ultrafast event and also as the signal of the subsequent optical parametric amplifiers.

In this setup, four optical imaging systems (OIS-1 to OIS-4) are used between the target object and the optical parametric amplifiers so that the plane of the target and those of the amplifiers are conjugated with each other. OIS-1 images the target on NCOPA-1 by optical magnification to match the spatial 
bandwidth of the amplifier, thus optimizing the imaging quality. OIS- 2 to OIS- 4 are used for $1 \times$ relay imaging. Four pieces of 0.5 -mm-thick and 29.2 deg-cut $\beta$-BBO crystals operate for OPA under type-I phase matching. In every amplifier, the pump and the signal are arranged with a small intersection angle $(\sim 2 \mathrm{deg})$ inside the $\beta$-BBO crystals so that the generated idler deviates spatially from both of them. Here the delay time between them can be independently adjusted with the DLs (DL-1 to DL-4). Meanwhile, a lens is used in every idler path to image the $\beta$ BBO crystal onto a CCD camera for image quality optimization. Using four noncollinear optical parametric amplifiers, we implement single-shot imaging with a frame number of four. The temporal resolution is about $50 \mathrm{fs}$, estimated using the idler pulse duration measured with our homemade SPIDER, whereas the spatial resolutions depend on the spatial bandwidth of the optical parametric amplifiers and the optical imaging system including OIS-1 to OIS-4 and L1 to L4. In principle, the minima of the frame intervals depending on the DL-1 to DL-4 can be at the femtosecond level. However, an excessively small frame interval will result in a strong overlap of the information between the adjacent frame images, so little effective information can be available. In the following experiments, the frame interval $\Delta t$ is chosen to be longer than the idler pulse duration or the resolved time- to avoid the information overlaps of adjacent frame images.

\section{Results and Discussion}

\subsection{System Characterization and Calibration}

In addition to the encouraging temporal resolution, using a femtosecond laser pulse as the pump to realize OPA imaging has two unique advantages: high OPA gain and spatial bandwidth. The former allows the use of a weak sampling signal, whereas the latter means a high spatial resolution. Both of these advantages are pump intensity dependent: a stronger pump pulse can bring a higher OPA gain and a larger spatial bandwidth. The available pump intensity is mainly limited by the laser-induced damage of the OPA crystal, which also depends on the pump pulse duration: the shorter the pump duration is, the higher the available pump intensity is. For femtosecond pulses, the pump intensity can be as high as hundreds of $\mathrm{GW} / \mathrm{cm}^{2} .{ }^{33}$ In contrast, nanosecond pulses usually have an intensity below $10 \mathrm{GW} / \mathrm{cm}^{2}$. In this experiment, the pump level was set at $15 \mathrm{GW} / \mathrm{cm}^{2}$, and the OPA gain was about 30 . As a result, the conversion efficiency from the pumps to the idlers can be estimated at $0.26 \%$. The idlers had their bandwidths of about $24 \mathrm{~nm}$, and their central wavelengths were around $800 \mathrm{~nm}$ but varied slightly with the relative time delays between the pumps and the signals. When we tested the system using a USAF 1951 resolution pattern, the smallest resolvable feature was measured at $36 \mathrm{lp} / \mathrm{mm}$, which agreed well with our calculation (detailed in Note S1 in the Supplementary Material). In FINCOPA, the measurable time window depends on the pulse duration of the sampling pulse: the longer the stretched signal pulse duration is, the larger the sampling window is. However, the excessively large pulse duration leads to a considerably weak signal level, which can result in a strong parametric fluorescence background for OPA. Here a stretched pulse duration of 50 ps was chosen according to the lifetime of our first target: air plasma grating.
Before our ultrafast imaging, two steps should be taken for spatial and temporal calibrations. The first step was to calibrate the transverse positions of the four CCDs and the magnification of the optical imaging system, which had been carried out by capturing simultaneously the test patterns with the CCDs. As for the second step, we defined the initial time, or "time zero," as the moment when the signal interacted with the pump of NCOPA-1 (pump-1). This moment could be changed by adjusting the time delay of the first pump pulse via DL-1. Correspondingly, the time zero positions of NCOPA-2, NCOPA-3, and NCOPA-4 could be fixed by tuning the time delays of their pumps so that the signal amplified by NCOPA-1 was also maximized by NCOPA-2, NCOPA-3, and NCOPA-4 at the same time. The framing image, which was carried by the idler pulse (idler-1) and captured with camera CCD-1, was named the first image. Sequentially, the other three idler images with CCD-2, CCD-3, and CCD-4 were the second, third, and fourth images, respectively. Their moments relative to the time zero were adjusted with DL-2, DL-3, and DL-4 to change the time delays of the pump beams, i.e., pump-2, pump-3, and pump-4.

\subsection{Single-Shot Ultrafast Imaging of an Air Plasma Grating}

To check the performance of FINCOPA, we constructed an air plasma grating as our first target because it has adjustable structures with a spatial period down to $10 \mu \mathrm{m}$ and a lifetime of picoseconds,${ }^{34}$ thereby requiring a subpicosecond resolved time with a micrometer-level spatial resolution. The air plasma grating was excited by two noncollinear $800-\mathrm{nm}$ ultrashort pulses using a noncollinear interferometer (NCI) [see Fig. 7(a) in the Appendix]. The total energy of the exciting pulse was $2.4 \mathrm{~mJ}$, and the focal length of the lens $(\mathrm{L})$ was $250 \mathrm{~mm}$. The grating period could be tuned by varying the crossing angle of the two beams $(2 \alpha)$. Because these two beams also came from the output of our Ti:S laser system, the target grating could be synchronized accurately with the pumps and signals.

Figure 2(a) presents the target grating structure for $2 \alpha=$ 3.8 deg, whereas Fig. 2(b) shows the one-dimensional intensity profile recorded along the vertical white line in Fig. 2(a). We find that the grating has a modulation period of $12 \mu \mathrm{m}$ corresponding to a groove density of about $83 \mathrm{lp} / \mathrm{mm}$ along the vertical direction. According to our design, the NCOPAs can resolve spatial structures with a spatial frequency as high as $36 \mathrm{lp} / \mathrm{mm}$, so a magnification of $3 \times$ was chosen in OIS-1 to image the target onto the NCOPAs for the $83 \mathrm{lp} / \mathrm{mm}$ grating.

The two excitation pulses for the air plasma were from a 1-kHz Ti:S laser system together with a single pulse selector. In the absence of the single pulse selector, the event operated repeatedly at $1 \mathrm{kHz}$, so it was recorded by the pump-probe method, which was used to experimentally validate our next single-shot subsequent frame images. We implemented the pump-probe to record the evolution of the plasma grating with NCOPA-1 and CCD-1, which, as shown in Fig. 2(c), includes 16 subpictures. Each of the subpictures has a vertical white line for the calibration of the spatial position along the horizontal direction. In every subpicture, the plasma grating propagates from left to right. We defined the time zero as when the plasma crosses the white line in the first subpicture in Fig. 2(c), whereas the time delays of the other subpictures were marked by different values. Figure 2(d) presents the variation of the modulation [see Eq. (4) in the Appendix] versus time delay, from which we 

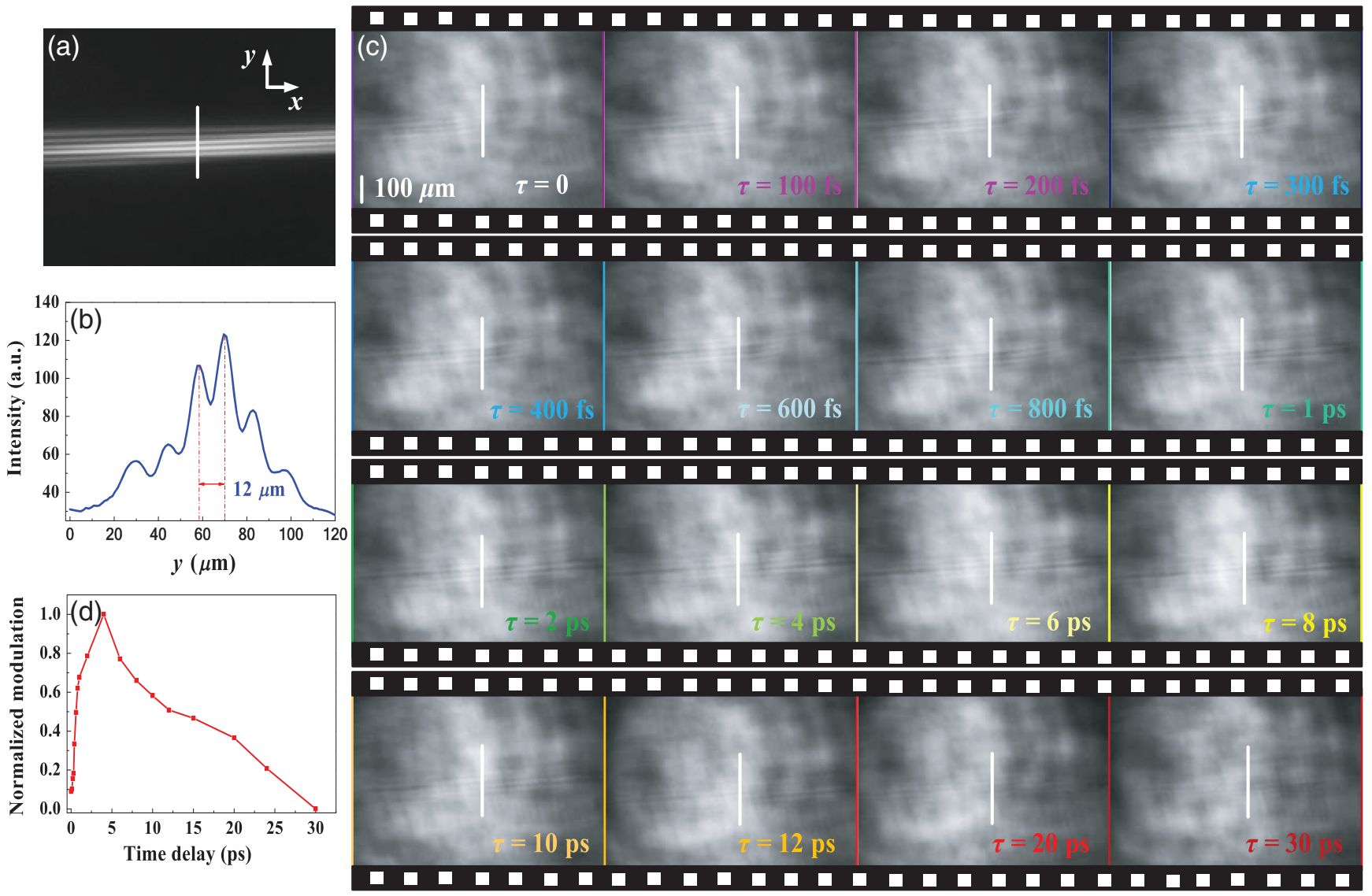

Fig. 2 (a) Static image of a plasma grating. The propagation of the pump pulse is in the $x$ direction. (b) The recorded one-dimensional intensity profile along the vertical white line in (a). (c) 16 idler images of the grating at 16 different moments were recorded by the pump-probe method. (d) The modulation evolution of the grating across the white lines of each image versus time delay.

can estimate that after the pump pulse passes through the white line the plasma grating becomes monotonously stronger but begins to disappear from 4 ps later.

For single-shot framing imaging, we set a single pulse selector at the exit of the Ti:S system to generate a single-shot plasma grating. Figure 3 presents four groups, each of which includes four movie frames of the grating obtained with the FINCOPA system, which have well resolved the grating (see Video S1). In Fig. 3(a), time intervals between the adjacent idler images are $100 \mathrm{fs}$. In other words, FINCOPA is operated at the frame rate of 10 Tfps (see Video S2). Figure 3(a) also shows that the streaks of the plasma grating from the left to the right become gradually visible over time, which means that the electron plasma density increases monotonously from 0 to 300 fs. From Eq. (1), we can obtain the streak modulations along the white lines in every image. As shown in Fig. 3(e), the modulation increases monotonously with the time delay. The frame intervals depend only on the four independent pump delays and can be freely adjusted. Figure 3(b) presents four images at 0, 200, 400, and $600 \mathrm{fs,} \mathrm{or}$ with a frame interval of $200 \mathrm{fs}$ (see Video S3). The streaks of the plasma grating become increasingly clearer, which can be verified by the variation of the modulation along the white lines, as shown in Fig. 3(f). Based on the phenomena in Figs. 3(e) and $3(\mathrm{f})$, we increased the frame interval up to $1 \mathrm{ps}$ and moved the captured moment of NCOPA-1 from the time zero to 1 ps (see Video S4). The four images and the modulation curve are shown in Figs. 3(c) and 3(g), respectively. These figures reflect that the trends of evolution of the plasma grating, shown in Figs. 3(a) and 3(b), are further extended. In Fig. 3(d), we show the captured images of the grating at 5, 8, 20, and 30 ps (see Video S5). The visibility of the streaks decreases with time, which means that the plasma grating begins to gradually disappear from 5 to 30 ps. As a result, unlike those in Figs. 3(e)-3(g), the modulation in Fig. 3(h) decreases over time.

To gather all the data from Figs. 3(e)-3(h), we obtained the time courses of normalized modulation of the grating across the white lines of each image [blue marks in Fig. 4(a)]. The red marks correspond to Fig. 2(d), obtained by the pump-probe method. Comparing the blue marks with the red marks, we find that the evolution of the plasma grating from the two methods well coincides, which demonstrates the validity of FINCOPA.

When $2 \alpha=2.5 \mathrm{deg}$, the plasma grating period became $\sim 18 \mu \mathrm{m}$ (i.e., a groove density of $56 \mathrm{lp} / \mathrm{mm}$ ). We captured the structure information with a magnification ratio of $3 \times$ in OIS-1. The imaging area was $0.94 \mathrm{~mm} \times 0.94 \mathrm{~mm}$. In addition, we reduced the magnification ratio to $2 \times$, which was sufficient to distinguish the grating structure but increased the imaging area to $1.32 \mathrm{~mm} \times 1.32 \mathrm{~mm}$. We repeated the experiments for Fig. 3 for $2 \alpha=2.5 \mathrm{deg}$ rather than $3.8 \mathrm{deg}$, and thus obtained the results as shown in Fig. 4(b). The results show a good coincidence of the normalized modulations between by pumpprobe method and by the FINCOPA method. We also paid 

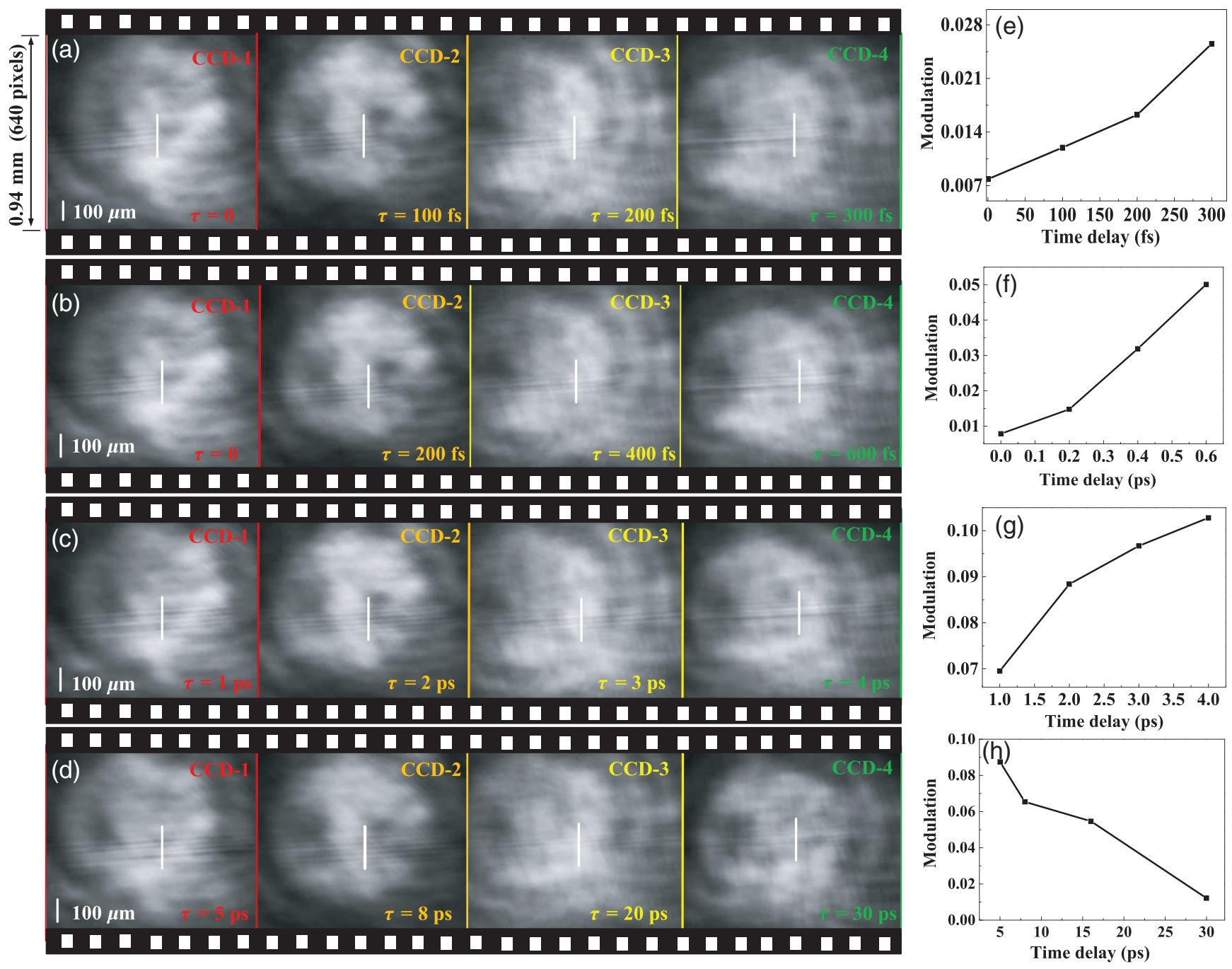

Fig. 3 Four images recorded in the single-shot mode at four moments: (a) 0, 100, 200, and 300 fs; (b) 0, 200, 400, and 600 fs; (c) 1, 2, 3, and 4 ps; and (d) 5, 8, 20, and 30 ps. The time-dependent modulations of the grating across the white lines are presented in $(\mathrm{e})-(\mathrm{h})$, respectively. (For the whole evolutionary process over time for plasma grating, see Video S1, MP4, 349 kB [URL: https:// doi.org/10.1117/1.AP.2.5.056002.1]; (a) see Video S2, MP4, 79 kB [URL: https://doi.org/10.1117/ 1.AP.2.5.056002.2]; (b) see Video S3, MP4, 82 kB [URL: https://doi.org/10.1117/1.AP.2.5. 056002.3]; (c) see Video S4, MP4, 103 kB [URL: https://doi.org/10.1117/1.AP.2.5.056002.4]; (d) see Video S5, MP4, 116 kB, [URL: https://doi.org/10.1117/1.AP.2.5.056002.5].

attention to the spatial evolution of the grating along its propagation direction. From the $4 \times 4$ framing images, we obtained the modulation index versus spatial coordinate along its propagation direction at different values of $\tau$, e.g., $0.8,1,2$, and $4 \mathrm{ps,}$ as shown in Fig. 5(a). The peak of the modulation moves toward the right with increasing $\tau$, which is explained by the fact that the pump pulse pair propagated from left to right. Because the plasma grating is a low-intensity-modulated object, the measured image contrast is relatively low. Using spatial filtering, we removed the background and increased imaging contrast (see Fig. S3 in the Supplementary Material).

Another observed phenomenon was that the peak values of the modulations decreased with the distance from the center along the $x$ direction. Figure 5(b) presents the modulation evolutions of the grating versus time from 0 to $30 \mathrm{ps}$ at four positions [see Fig. 5(c)] along the $x$ direction (i.e., $x=-15,-60,-90$, and $-500 \mu \mathrm{m}$ ). All of the positions show a similar evolution of the modulation, but the maxima decrease with a shift in the position from the center to the left. Thus Fig. 5(b) implies an $x$ dependence of the grating modulation, which may result from the $x$ dependence in exciting pulse intensity. To further verify this speculation, we estimated the evolution of the exciting pulse intensity along the $x$ direction. In our configuration of excitation, each of the pulse pairs had a pulse energy of $\sim 1 \mathrm{~mJ}$. Focused by a lens with a focal length of $150 \mathrm{~mm}$, the spot size $\omega_{0}$ was estimated to be at $7.64 \mu \mathrm{m}$. Setting our system so that the focal position corresponds to the time zero position, or the central line [marked as the white line in Fig. 5(c)], the $x$-dependent beam size is 

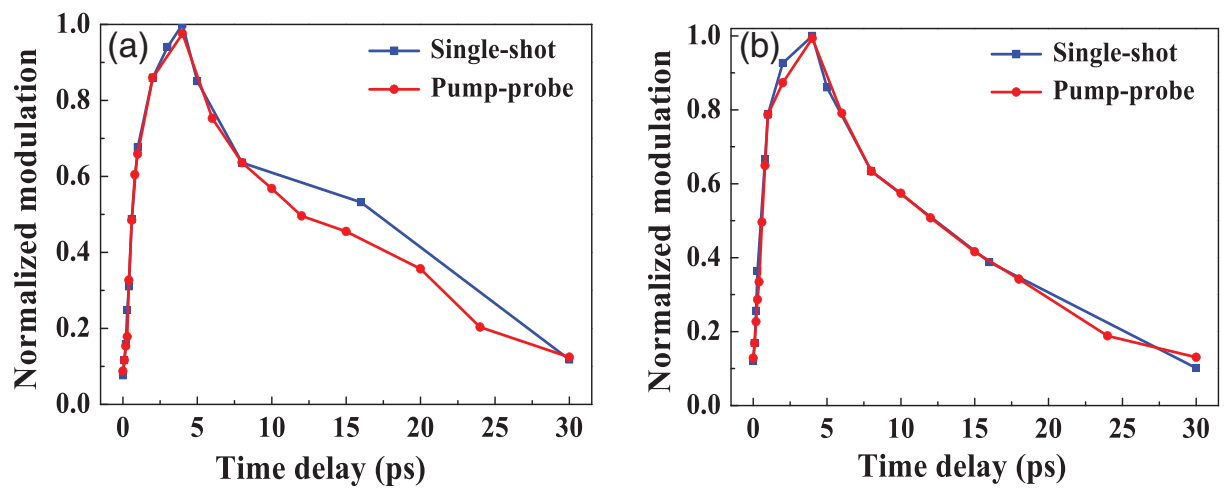

Fig. 4 Normalized modulations of the plasma grating across the white lines of each image versus time from 0 to 30 ps obtained by the pump-probe method (red marks) or by single-shot framing imaging (blue marks) for a magnification ratio of (a) $3 x$ and (b) $2 x$.
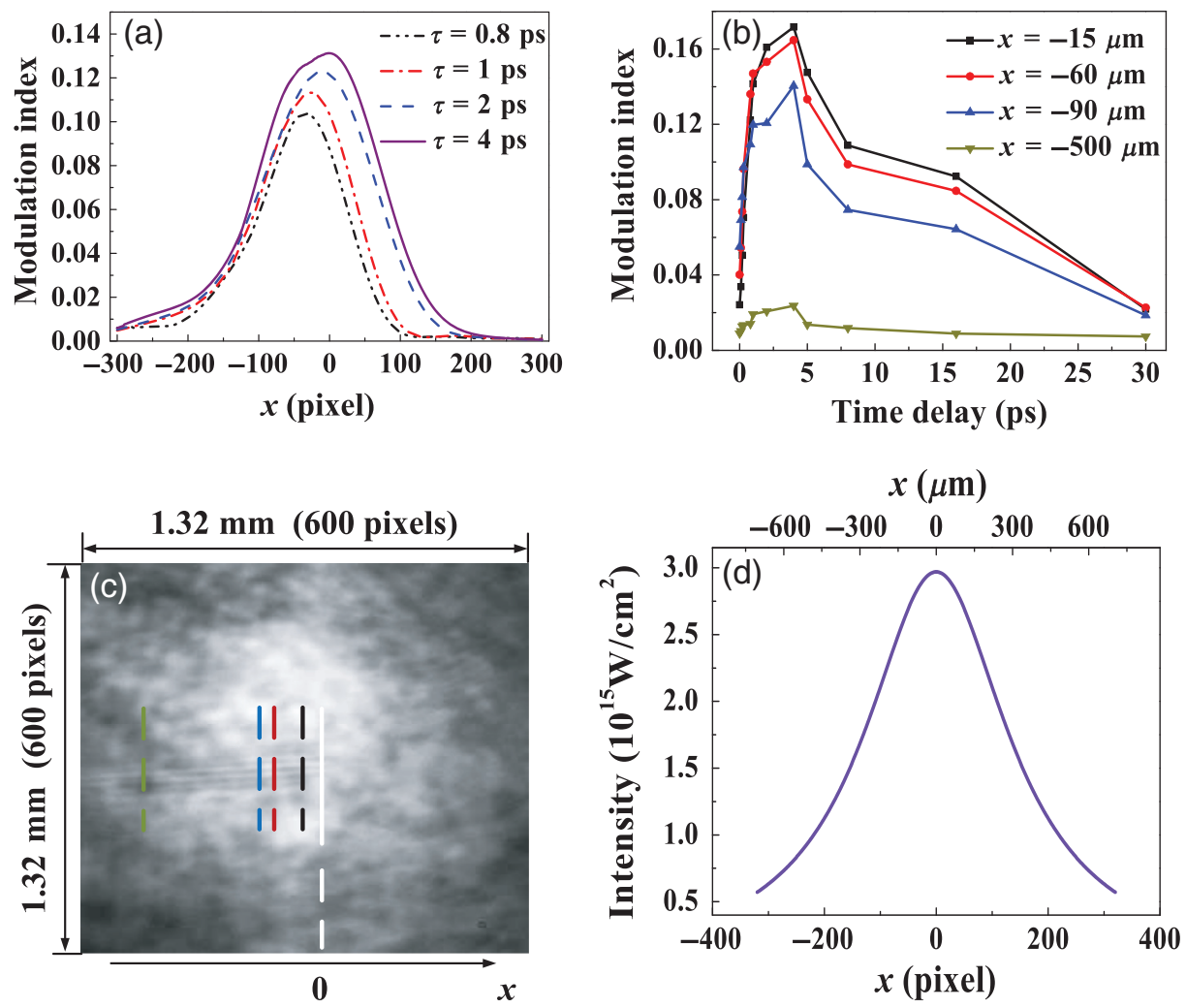

Fig. 5 (a) The modulation index versus the spatial coordinate; (b) the modulation index versus time at four different positions shown in (c); and (d) the evolution of exciting pulse intensity along the $x$ direction.

$\omega(x)=\omega_{0} \sqrt{1+\left(x / l_{R}\right)^{2}}$,

where Rayleigh length is $l_{R}=\pi \omega_{0}^{2} / \lambda$. And the $x$-dependent pulse intensity can be determined by

$I(x)=\frac{E_{p}}{\pi \omega^{2}(x) \tau_{p}}$, where $E_{p}$ and $\tau_{p}$ are the pulse energy and duration, respectively. From Eqs. (1) and (2), we obtained the evolution of the exciting pulse intensity along the $x$ direction, as shown in Fig. 5(d), in which the exciting pulse intensity decreases monotonously but symmetrically when the position is shifted away from the focal spot.

The measurements of the plasma grating structures verify that the FINCOPA imaging system can observe the grating structures at the micrometer level. The single-shot optical imaging for temporal evolutions of the plasma has been confirmed 
experimentally by those with the pump-probe method, which reveals that an interval time of $100 \mathrm{fs}$ is sufficient for FINCOPA to resolve the plasma evolution.

\subsection{Single-Shot Ultrafast Imaging of a Rotating Optical Field}

To further check the excellent temporal properties of our FINCOPA, we had successfully taken single-shot imaging for a $20-\mathrm{Hz}$ ultrafast rotating optical field with a rotation rate beyond 10 trillion radians per second (Trad/s). This event was generated with the setup shown in Fig. 7(b) in the Appendix, including an asymmetric Michelson interferometer and a spiral phase generator (SPG). ${ }^{35}$ Its low repetition rate $(20 \mathrm{~Hz})$ means that this kind of optical field can be boosted up to extremely high power (e.g., tens of terawatts, even higher). However, for a laser system, a low repetition rate usually comes with a large shot-shot fluctuation of its output pulses so that the pump-probe method may result in a considerable measurement inaccuracy. The target optical field was generated through overlapping two chirped vortex pulses with different topological charges $\pm l$ and a time delay $\delta t$. If tuning the time delay of a pair of 3 ps chirped pulses with $l= \pm 1$ to be $1 \mathrm{ps}$, the optical field was rotated with an angular frequency difference $\Delta \omega$ of $\sim 27 \mathrm{Trad} / \mathrm{s}$ (i.e., a rotating cycle of $466 \mathrm{fs}$ ). The corresponding rotating velocity $\Omega=13.5 \mathrm{Trad} / \mathrm{s}$ was faster than the highest rotating velocity of $11.9 \mathrm{Trad} / \mathrm{s}$ in Ref. 34. FINCOPA imaged this event with a frame interval $\Delta t$ of $66.7 \mathrm{fs}$ (i.e., a frame rate of 15 Tfps; see Video S6). Figure 6 shows the field rotating an angle of $\sim 0.9 \pi \mathrm{rad}$ within $200 \mathrm{fs}$. The rotating velocity $\Omega$ was calculated to be $14 \mathrm{Trad} / \mathrm{s}$, closely matching the theoretical value. As the optical field can rotate in the terahertz frequency region, this will be of great interest for laser plasma-based accelerators ${ }^{36,37}$ and strong terahertz radiations. ${ }^{38}$

It is interesting to explore the ability of our FINCOPA for microscopic imaging. To preliminarily explore this idea, we used a $20 \times$ microscope objective lens (Olympus, PLN20X) in OIS-1 for optical magnification. A transient target with microstructures is desirable, like a laser-plasma grating with a spatial period of some micrometers. However, according to our experiment, this kind of grating has too small of a modulation to be recorded by FINCOPA. Consequently, we used a static grating with a density of $300 \mathrm{lp} / \mathrm{mm}$ as a target instead of the dynamic grating. The idler image, captured with CCD-1, can distinctly exhibit the grating structure (details in Note $\mathrm{S} 2$ in the Supplementary Material). It is worth noting that there is no movement or deflection in the ultrafast image acquisition, so the spatial resolution of FINCOPA is equivalent to the static resolution. Microscopic ultrafast optical imaging using FINCOPA is well within reach.

\section{Conclusions}

We have realized single-shot ultrafast framing imaging by noncollinear OPA (FINCOPA). Using a laser-induced air plasma grating as a target, FINCOPA has experimentally recorded 50 -fs-resolved frame images with an effective frame rate of 10 Tfps. Moreover, FINCOPA has been used to successfully visualize an ultrafast rotating (13.5 Trad/s) optical field with an effective frame rate of 15 Tfps. To our knowledge, this work has provided the highest experimental record of the effective frame rate with the temporal resolution of $50 \mathrm{fs}$. FINCOPA has a high spatial resolution thanks to the large spatial bandwidth of the used optical parametric amplifiers. Using a $3 \times$ optical magnification ratio, the spatial resolution has been confirmed beyond $83 \mathrm{lp} / \mathrm{mm}$ with an imaging area of about $0.94 \mathrm{~mm} \times$ $0.94 \mathrm{~mm}$. If the magnification becomes $20 \times$, the spatial resolution can be beyond $300 \mathrm{lp} / \mathrm{mm}$ for an area of $0.2 \mathrm{~mm} \times 0.5 \mathrm{~mm}$, which means that FINCOPA is capable of ultrafast microscopic imaging.

In FINCOPA, the temporal resolution depends only on the trigger pulse duration, whereas the spatial resolution is determined mostly by the spatial bandwidth of the optical parametric amplifiers and the OIS. The frame interval can be flexibly adjusted from a few femtoseconds to tens of picoseconds with femtosecond accuracy for different ultrafast events. Ultimately, the frame number is limited by the ratio of the sampling pulse duration to the pump pulse duration. However, the laser pulses with durations of tens of femtoseconds are available from many commercial lasers. These pulses can be stretched to tens of nanoseconds with a stretching factor beyond $10^{5}$. Accordingly, the ratio of the sampling pulse duration to the pump pulse duration can be as large as $10^{5}$, which satisfies most scientific studies. In practice, the frame number is restricted by the output power of the used laser source, which limits the available number of the optical parametric amplifiers. All of the above analyses show that FINCOPA has much space for future improvement. For example, the temporal resolution can be improved using the trigger pulses with shorter temporal durations, whereas the frame number can be increased with more powerful lasers.

In summary, combining outstanding spatial and temporal resolutions with an ultrahigh frame rate, FINCOPA is a powerful single-shot ultrafast optical imaging technique for accurate temporal and spatial observations of single transient events

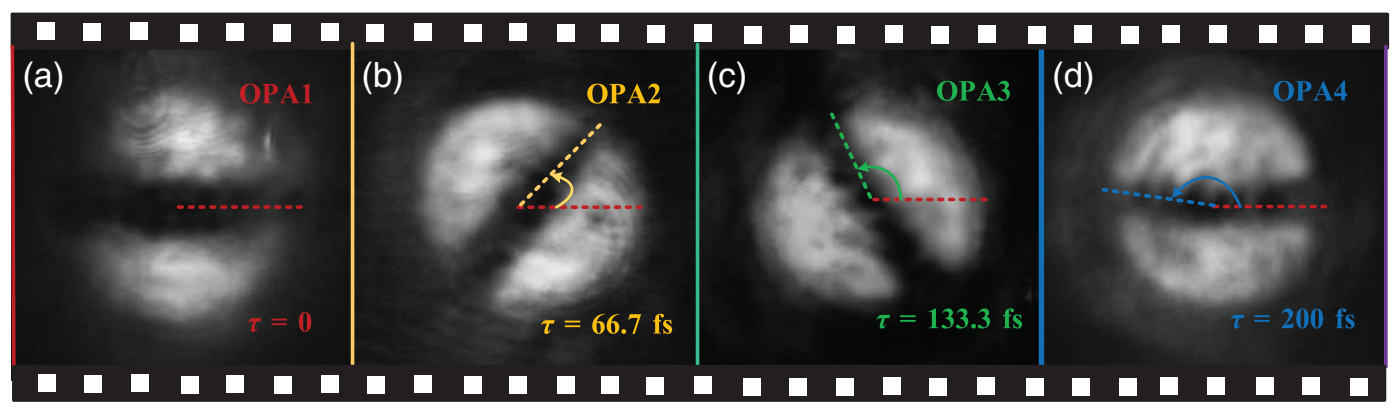

Fig. 6 (a)-(d) Four frame images of the ultrafast rotating optical field recorded in the single-shot mode at 15 Tfps (Video S6, MP4, 47 kB [URL: https://doi.org/10.1117/1.AP.2.5.056002.6]). 

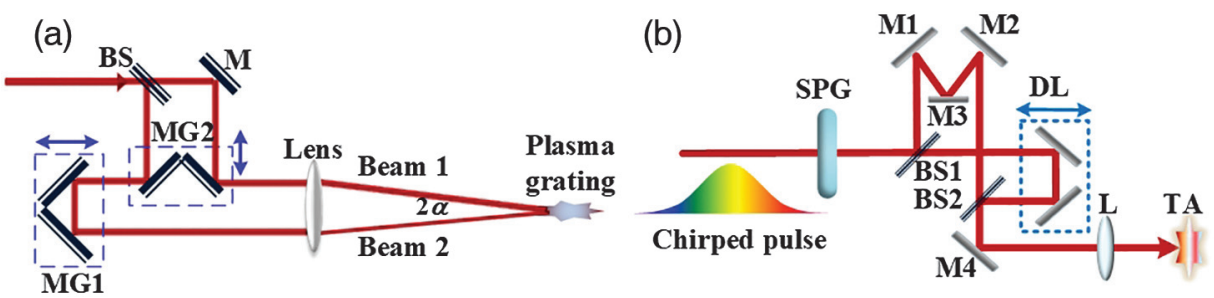

Fig. 7 (a) Setup of the plasma grating generation, an NCl. M, mirror; MG1 and MG2, mirror groups; and BS, beam splitter. (b) Setup of the ultrafast rotating optical lattice generation. ${ }^{35}$ SPG, spiral phase generator; M1 to M4, mirrors; BS1 and BS2, beam splitters; and DL, delay line.

occurring in the femtosecond region, such as atomic or molecular dynamics in photonic materials, ${ }^{39}$ plasma physics, and ultrafast laser ablation for precise machining and manufacturing.

\section{Appendix: Methods}

\subsection{Generation of a Plasma Grating and an Ultrafast Rotating Optical Field}

The first target is a plasma grating, which is formed by focusing a pair of femtosecond pulses with a small crossing angle $2 \alpha$. The grating period is

$\Lambda=\lambda /[2 \sin (\alpha)]$

As the angle $2 \alpha$ becomes larger, the number of streaks increases, whereas the grating period $\Lambda$ decreases. Figure 7(a) shows the plasma grating excited by two noncollinear ultrashort pulse beams using an NCI. The key to this design is the two mirror groups: MG1 and MG2. Both groups consist of two mirrors set orthogonally to each other. In this design, a 50/50 BS is first used to split the input pulse into two beams (beam 1 and beam 2), and a lens symmetrically focuses both beams. MG1 is responsible for the time delay between beam 1 and beam 2, whereas MG2 tunes the angle $2 \alpha$. Moving MG1 to realize accurate temporal synchronization will not change the spatial distributions of beam 1 and beam 2 or the spatial overlap between the two beams. Moreover, moving MG2 will result in parallel but symmetrical shifts of the beams away from or toward the optical axis of the lens, thereby changing the angle $2 \alpha$. However, moving MG2 will never change the spatial overlap and the time delay between the two beams, which helps to obtain a high stability and high repeatability in our experiments.

For convenience in characterizing the evolution of the plasma grating from the frame images, we calculate the grating modulation, which is associated with the electron density, recombination coefficient, and refractive index of the plasma ${ }^{40}$ by

$M=\frac{I_{\max }-I_{\min }}{I_{\max }+I_{\min }}$,

where $I_{\max }$ and $I_{\min }$ are the maximum and minimum intensities of the images, respectively.

The second target is an ultrafast rotating optical field. ${ }^{35}$ As shown in Fig. 7(b), a chirped pulse, which is converted into a vortex pulse by an SPG, passes through an asymmetric Michelson interferometer where there are three mirrors (M1 to M3) in one arm, while there are two mirrors (for time delay) in the other. This asymmetrical design allows the two output pulses from the Michelson interferometer to have topological charges $\pm l$, time delay $\delta t$, and the same spatial amplitude $E_{0}$. In this way, the rotating velocity can be expressed as

$\Omega=\frac{\mathrm{d} \theta}{\mathrm{d} t}=\frac{\Delta \omega}{\Delta l}=\frac{C \delta t}{\Delta l}$,

where $C=4 \ln (2) / \tau^{2}\left[\left(\tau / \tau_{0}\right)^{2}-1\right]^{1 / 2}$ is the chirp coefficient. It depends on the duration $\tau$ of the chirped pulse that is stretched from a transform-limited pulse with a duration of $\tau_{0} . \Omega$ is proportional to $\delta t$ but inversely proportional to $\tau$ and $\Delta l$. The rotating rate can be beyond $10 \mathrm{Trad} / \mathrm{s}$, which can be flexibly tuned with a mirror in the Michelson interferometer.

\section{Acknowledgments}

This work was supported partly by the National Natural Science Foundation of China (Nos. 61775142 and 61705132), the Shenzhen Basic Research Project on the subject layout (No. JCYJ20170412105812811), the Shenzhen Basic Research Projects (Nos. JCYJ20170412105812811, JCYJ20190808164007485, and JCYJ20190808115601653), and the Natural Sciences and Engineering Research Council of Canada (Nos. RGPIN-2017-05959 and RGPAS-507845-2017).

There are no conflicts of interest.

\section{References}

1. M. Garcia-Lechuga et al., "Femtosecond laser ablation of dielectric materials in the optical breakdown regime: expansion of a transparent shell," Appl. Phys. Lett. 105, 112902 (2014).

2. R. Betti and O. A. Hurricane, "Inertial-confinement fusion with lasers," Nat. Phys. 12, 435-448 (2016).

3. A. Couairon and A. Mysyrowicz, "Femtosecond filamentation in transparent media," Phys. Rep. 441, 47-189 (2007).

4. K. Nakagawa et al., "Sequentially timed all-optical mapping photography (STAMP)," Nat. Photonics 8, 695-700 (2014).

5. P. Hockett et al., "Time resolved imaging of purely valenceelectron dynamics during a chemical reaction," Nat. Phys. 7, 612-615 (2011).

6. G. Herink et al., "Real-time spectral interferometry probes the internal dynamics of femtosecond soliton molecules," Science 356, 50-54 (2017).

7. X. Liu and Y. Cui, "Revealing the behavior of soliton buildup in a mode-locked laser," Adv. Photonics 1(1), 016003 (2019).

8. C. Y. Wong et al., "Eletronic coherence lineshapes reveal hidden excitonic correlations in photosynthetic light harvesting," Nat. Chem. 4, 396-404 (2012).

9. J. Liang et al., "Single-shot real-time video recording of photonic Mach cone induced by a scattered light pulse," Sci. Adv. 3, e1601814 (2017). 
10. A. Velten et al., "Recovering three-dimensional shape around a corner using ultrafast time-of-flight imaging," Nat. Commun. 3, 745 (2012).

11. A. H. Zewail, "Four-dimensional electron microscopy," Science 328, 187-193 (2010).

12. J. Liang and L. V. Wang, "Single-shot ultrafast optical imaging," Optica 5(9), 1113-1127 (2018).

13. J. Liang, "Punching holes in light: recent progress in singleshot coded-aperture optical imaging," Rep. Prog. Phys. (accepted manuscript) (2020).

14. P. W. W. Fuller, "An introduction to high speed photography and photonics," Imaging Sci. J. 57, 293-302 (2009).

15. C. Yang et al., "Optimizing codes for compressed ultrafast photography by the genetic algorithm," Optica 5(2), 147-151 (2018).

16. L. Gao et al., "Single-shot compressed ultrafast photography at one hundred billion frame per second," Nature 516, 74-77 (2014).

17. J. Liang, L. Zhu, and L. V. Wang, "Single-shot real-time femtosecond imaging of temporal focusing," Light Sci. Appl. 7, 42 (2018).

18. T. King et al., "Picosecond-resolution phase-sensitive imaging of transparent objects in a single shot," Sci. Adv. 6(3), eaay6200 (2020).

19. K. Goda, K. K. Tsia, and B. Jalali, "Serial time-encoded amplified imaging for real-time observation of fast dynamic phenomena," Nature 458, 1145-1149 (2009).

20. N. H. Matlis, A. Axley, and W. P. Leemans, "Single-shot ultrafast tomographic imaging by spectral multiplexing," Nat. Commun. 3, 1111 (2012).

21. Z. Li et al., "Single-shot tomographic movies of evolving lightvelocity objects," Nat. Commun. 5, 3085 (2014).

22. T. Suzuki et al. "Sequentially timed all-optical mapping photography (STAMP) utilizing spectral filtering," Opt. Express 23(23), 30512-30522 (2015).

23. M. Tamamitsu et al., "Design for sequentially time all-optical mapping photography with optimum temporal performance," Opt. Lett. 40(4), 633-636 (2015).

24. T. Suzuki et al., "Single-shot 25-frame burst imaging of ultrafast phase transition of $\mathrm{Ge}_{2} \mathrm{Sb}_{2} \mathrm{Te}_{5}$ with a sub-picosecond resolution," Appl. Phys. Express 10, 092502-4 (2017).

25. A. Ehn et al., "FRAME: femtosecond videography for atomic and molecular dynamics," Light Sci. Appl. 6, e17045 (2017).

26. G. Chen et al., "All-optical coaxial framing photography using parallel coherence shutters," Opt. Lett. 42(3), 415-418 (2017).

27. G. Chen, J. Li, and J. Li, "Femtosecond multiframe digital holography with parallel coherence shutters," in Digital Hologr. and $3 D$ Imag., Bordeaux, France, p. Th4B.1, (2019).

28. Y. Lu et al., "Compressed ultrafast spectral-temporal photography," Phy. Rev. Lett. 122, 193904 (2019).

29. J. Moon et al., "Single-shot imaging of microscopic dynamic scenes at $5 \mathrm{THz}$ frame rates by time and spatial frequency multiplexing," Opt. Express 28(4), 4463-4474 (2020).

30. X. Zeng et al., "High resolved non-collinear idler imaging via type-II angular noncritical phase matching," IEEE Photonics Technol. Lett. 28(23), 2685-2688 (2016).

31. W. Li et al., "Ultrafast laser pulse synchronization," in Coherence and Ultrashort Pulse Laser Emission, F. J. Duarte, Ed., InTech, Rijeka, Croatia (2010).

32. P. M. Vaughan and R. Trebino, "Optical-parametric-amplification imaging of complex objects," Opt. Express 19(9), 8920-8929 (2011).

33. P. S. Banks, M. D. Feit, and M. D. Perry, "High intensity thirdharmonic generation," J. Opt. Soc. Am. B 19(1), 102-118 (2002).

34. X. Yang et al., "Femtosecond laser pulse energy transfer induced by plasma grating," Appl. Phys. Lett. 97, 071108 (2010).

35. X. Zeng et al., "Generation and imaging of a tunable ultrafast intensity-rotating optical field with a cycle down to femtosecond region," High Power Laser Sci. Eng., 8, e3 (2020).

36. Y. Shi et al., "Magnetic field generation in plasma waves driven by copropagating intense twisted lasers," Phys. Rev. Lett. 121, 145002 (2018).
37. J. Vieira et al., "Amplification and generation of ultra-intense twisted laser pulses via stimulated Raman scattering," Nat. Commun. 7, 10371 (2016).

38. E. Nanni et al., "Terahertz-driven linear electron acceleration," Nat. Commun. 6, 8486 (2015).

39. K. Lin et al., "Spatiotemporal rotational dynamics of laser-driven molecules," Adv. Photonics 2(2), 024002 (2020).

40. A. Jarnac, "Study of laser induced plasma grating dynamics in gases," Opt. Commun. 312, 35-42 (2014).

41. M. Vasilyev, N. Stelmakh, and P. Kumar, "Estimation of the spatial bandwidth of an optical parametric amplifier with plane-wave pump," J. Mod. Opt. 56, 2029-2033 (2009).

42. L. V. Sokolov, M. I. Kolobov, and L. A. Lugiato, "Quantum fluctuations in traveling-wave amplification of optical images," Phys. Rev. A. 60(3), 2420-2430 (1999).

43. X. Zeng et al., "High spatial resolved idler image with a compact non-collinear optical parametric amplifier using a CW laser as signal," IEEE Photonics J. 7(6), 6804107 (2015).

Xuanke Zeng received his $\mathrm{PhD}$ in optics engineering from Shenzhen University. He is now a postdoc at Shenzhen University. His main research interests are ultrafast optics and ultrafast imaging.

Shuiqin Zheng received his $\mathrm{PhD}$ in optics engineering from Shenzhen University in 2019. He is now a postdoc at King Abdullah University of Science and Technology. His research interests include ultrafast laser technology, terahertz technology, and singular optics.

Yi Cai received his BS degree in physics from Sun Yat-sen University in 2003 and his PhD in optics from Shanghai Institute of Optics and Fine Mechanics at the Chinese Academy of Sciences in 2010. He is an assistant professor at Shenzhen University. He is the author of more than 20 journal papers. His current research interests include nonlinear optics, ultrafast laser technology, and ultrafast imaging.

Qinggang Lin received his master's degree in electronic science and technology from Shenzhen University of China. He is a $\mathrm{PhD}$ student at Shenzhen University. His main research interest is terahertz generation and detection.

Jinyang Liang is an assistant professor at the Institut National de la Recherche Scientifique of the University du Québec. His research focuses on the development of high-speed computational optical instruments for applications in biology and physics. He has published more than 50 journal and conference papers and holds 2 patents on ultrafast optical imaging technology. He was the recipient of the 2019 Young Scientist Prize from IUPAP, the 2019-2023 Research Scholarship from FRQS, and the 2017 Educational Award-Gold from Edmund Optics.

Xiaowei Lu received her BS degree in physics from Minzu University of China in 2006 and her MS and PhD degrees in optics and optical engineering from Shenzhen University in 2009 and 2012, respectively. She is an assistant professor at Shenzhen University. She is the author of more than 20 journal papers. Her current research interests include single-shot ultrafast imaging, holography, and optical image analysis and processing.

Jingzhen Li graduated from Tsinghua University and transferred from the Xi'an Institute of Optics and Precision Mechanics at Academia Sinica to Shenzhen University in 1994. He is a professor at Shenzhen University. He has been a vice president of the Committee for Optical Testing of COS and a vice president of the Committee for High-Speed Photography and Photonics of COS. He has been studying high-speed imaging and photonics for a long time. His main research interests currently include extreme-high-speed imaging with tubeless, rotating mirror cameras, and especially, all-optical ultrafast imaging. So far, he has published more 
than 200 papers and 10 books about optics, laser, and high-speed imaging, won six state-level science and technology awards, and twelve provincial and ministerial science and technology awards in China.

Weixin Xie is a professor and supervisor of $\mathrm{PhD}$ students at Shenzhen University. He has been the leader of the electronic subject consultative group of the National Natural Science Foundation of China. He is the author of more than 200 journal papers. His current research interests include signal processing and information processing, intelligent humancomputer interaction, image processing, and pattern recognition.
Shixiang Xu received his PhD in 1998 from Shanghai Institute of Optics and Fine Mechanics, China. He is a professor at Shenzhen University. Currently, he serves as a member of the Laser Professional Committee of the Chinese Optical Society and one of the councils of Guangdong Optical Society of China. In 2006, he was awarded the Shanghai Pujiang Talent Fund. To date, he has published more than 130 papers in peerreviewed journals and authorized more than 25 patents. His research interests include ultrashort pulse laser, ultrafast imaging, and pulsed terahertz optics. 